\title{
Biliary cholesterol secretion by the twinned sterol half-transporters ABCG5 and ABCG8
}

\author{
Henning Wittenburg and Martin C. Carey \\ Department of Medicine, Harvard Medical School, Gastroenterology Division, Brigham and Women's Hospital, \\ and Harvard Digestive Diseases Center, Boston, Massachusetts, USA \\ J. Clin. Invest. 110:605-609 (2002). doi:10.1172/JCI200216548.
}

The long wait for a cellular and functional definition of canalicular transporters for biliary cholesterol appears to be over. Articles in this issue of the JCI provide a fascinating account of the cellular itinerary of two murine ATP-binding cassette (ABC) halftransporters from the ribosome to the hepatocyte apical membranes (1) and show that the corresponding human genes, when overexpressed in transgenic mice, can greatly augment cholesterol secretion into bile (2).

This field was opened with the genetic solution $(3,4)$ to the enigma of a disease commonly known as sitosterolemia $(5,6)$. This autosomal recessive disorder of lipid metabolism is characterized by an accumulation of plant sterols, including sitosterol, in the plasma of patients with this disease and is therefore also known as phytosterolemia. Normally, the intestinal absorption of sitosterol and other unwanted plant sterols is dwarfed by the absorption of cholesterol, and the absorbed phytosterols are cleared from the body by highly efficient secretion into bile (5). However, patients with sitosterolemia dis-

\footnotetext{
Address correspondence to: Martin C. Carey, Brigham and Women's Hospital, Gastroenterology Division, 75 Francis Street, Boston, Massachusetts 02115, USA. Phone: (617) 732-5822; Fax: (617) 730-5807; E-mail: mccarey@rics.bwh.harvard.edu. Henning Wittenburg's present address is: Department of Medicine II, University of Leipzig, Leipzig, Germany.

Conflict of interest: No conflict of interest has been declared.

Nonstandard abbreviations used: ATPbinding cassette $(\mathrm{ABC})$; endoplasmic reticulum (ER); mammalian multidrug resistance (MDR); cholesterol saturation index (CSI).
}

play intestinal hyperabsorption of plant sterols and impaired biliary sterol secretion, leading to accumulation of phytosterols in the body (6). The metabolism of cholesterol is altered in the same manner, but to a lesser extent (5), rendering many patients hypercholesterolemic (6) and prone to xanthomas and premature atherosclerosis $(5,6)$. The dramatic consequences of this rare disease demonstrate the importance of a finely tuned mechanism for restricting the absorption and accumulation of ingested sterols. The underlying pathophysiological defects indicated that unraveling the molecular basis of sitosterolemia might shed light on important principles concerning intestinal sterol absorption as well as cholesterol secretion into bile.

Two independent groups $(3,4)$ identified mutations in sitosterolemia patients in either member of an adjacent pair of genes, $A B C G 5$ and $A B C G 8$, encoding $A B C$ transporters expressed in the liver and intestine $(3,4,7)$. Functional ABC transporters consist of 12 membrane-spanning domains and two ATP-binding sites, whereas ABCG5 and ABCG8 are examples of half-transporters containing only six transmembrane domains and one ATP-binding site. Therefore, both teams of investigators $(3,7)$ suggested that ABCG5 and ABCG8 function as a heterodimer to limit intestinal sterol absorption by effluxing sterols from enterocytes to small intestinal lumen and by promoting hepatic sterol secretion into bile. Still, in the absence of direct evidence, the function of ABCG5 and ABCG8 and their subcellular localization remained hypothetical until now.

\section{ABCG5 and ABCG8 half- transporters "hold hands" on their journey to the apical membrane} In an elegant series of cell culture experiments, Graf et al. (1) used epitope-tagged ABCG5 and ABCG8 to circumvent the unavailability of antibodies for the proteins and confirmed that mature ABCG5 and ABCG8 are expressed on the apical plasma membrane of polarized hepatocyte cell models $(5,6)$. Exit of the proteins from the endoplasmic reticulum (ER) requires coexpression of both $\mathrm{ABC}$ half-transporters, suggesting that formation of a heterodimer is necessary to target ABCG5 and ABCG8 to the apical plasma membrane. Further indirect support for the formation of ABCG5 and ABCG8 heterodimers came from colocalization of both halftransporters in the ER and plasma membrane, co-immunoprecipitation of both proteins, and finally, comparison of the post-translational modifications that the proteins undergo when they are expressed singly or together in transfected cells. These results led the authors to propose that ABCG5 and ABCG8 dimerize in the $\mathrm{ER}$, traffic together to the Golgi apparatus, and become targeted to the apical plasma membrane, where they function as an export pump for neutral sterols (1). This model readily explains the earlier finding that mutations in either $A B C G 5$ or $A B C G 8$ suffice to cause sitosterolemia $(3,4)$.

\section{Increased biliary cholesterol transport in mice overexpressing ABCG5 and ABCG8}

In the second study, $\mathrm{Yu}$ et al. (2) addressed the function of ABCG5 and ABCG8 by generating and characteriz- 


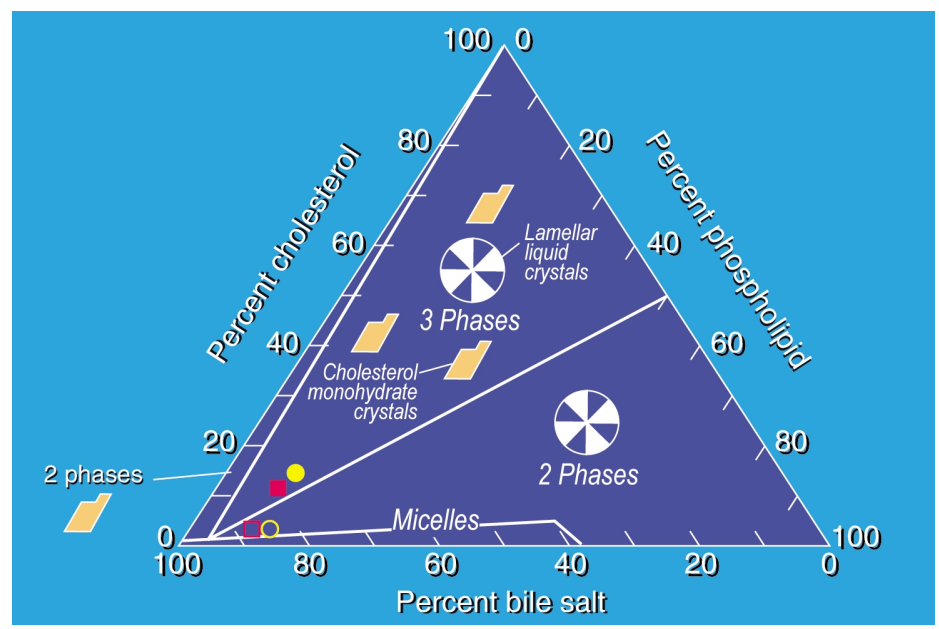

\section{Figure 1}

Hypothetical condensed phase diagram for gallbladder bile of mice that represents an estimate for an equimolar taurocholate-tauro- $\beta$-muricholate mixture $(11,12)$. The division of the phase diagram for gallbladder bile of $\sim 7.3 \mathrm{~g} / \mathrm{dL}$ lipids $\left(37^{\circ} \mathrm{C}\right)$ above the micellar zone is interpolated from published phase diagrams for both pure taurocholate and the pure hydrophilic bile salt tauroursodeoxycholate, each with natural (egg) phosphatidylcholine and cholesterol (11). However, it takes into consideration that $\beta$-muricholate (the taurine conjugate representing $50 \%$ of the bile salt pool in ref. 2) displays approximately half the solubility of ursodeoxycholate for cholesterol (8, 24). Above the small micellar zone are, from left to right, two-, three-, and two-phase regions, each of which contains mixed micelles and separated phases which are indicated schematically on the figure. Relative lipid compositions of Yu et al.'s (2) littermate control mice (open circles, females; open squares, males) plot just above the micellar zone, indicating that they are slightly supersaturated with cholesterol. However, biles of the transgenic animals (closed circles, females; closed squares, males) are markedly enriched in cholesterol (2), plotting within the three-phase zone, and display apparent CSIs of approximately 9 for females and 7 for males on this phase diagram. Note that a hypothetical line connecting control and transgenic bile extrapolates to the cholesterol apex of the triangle, a phenomenon not usually encountered in the murine lithogenic state (12). This implies that biliary phospholipid secretion was uncoupled from cholesterol secretion by ABCG5/ABCG8 overexpression, thereby limiting the molar ratio of cholesterol that could be solubilized by phospholipid at the canalicular level.

ing transgenic mice that overexpress both human proteins coordinately. Compared to littermates, transgenic mice display markedly elevated biliary cholesterol secretion rates (as evidenced by gallbladder cholesterol concentrations), increased fecal neutral sterol excretion, and markedly reduced plasma phytosterol levels. The lower cholesterol absorption in transgenic mice compared to littermates probably reflects both the activity of the overexpressed ABCG5 and ABCG8 in enterocytes and the animals' high rate of biliary cholesterol secretion. Since high phytosterol plasma levels occur in sitosterolemia, the low plasma phytosterol levels in the transgenic mice are consistent with the control of phytosterol absorption and biliary secretion being critically dependent on the ABCG5/ABCG8 transporter. In contrast, cholesterol absorption and plasma levels are only changed modestly, indicating that their controls are genetically more complex in that ABCG5 and ABCG8 may not be rate limiting in their regulation.

Cholesterol for increased biliary cholesterol secretion is supplied by augmented de novo cholesterol synthesis in $A B C G 5 / A B C G 8$ transgenic mice. Female transgenic mice also have lower plasma HDL cholesterol levels, perhaps because their biliary cholesterol secretion rates are even higher than those in males and outpace de novo cholesterol biosynthesis. Other differences between the sexes have been reported for numerous aspects of lipid metabolism in laboratory mice. Still, it is important to keep in mind that the transgenic founder animals in the present work derived from a mixed genetic background and were backcrossed to $\mathrm{C} 57 \mathrm{BL} / 6 \mathrm{~J} \times \mathrm{SJL} \mathrm{F}_{1}$ mice. Because of segregating alleles, all mice of this colony are genetically different from each other, possibly complicating the analysis of sex-specific phenotypes.
Future experiments of ABCG5/ABCG8 function in mice will clearly benefit from breeding the transgenes onto a defined genetic background.

\section{The surprising absence of cholesterol gallstones in transgenic animals}

Analysis of gallbladder biles from male and female transgenic mice revealed markedly increased cholesterol concentrations compared to littermate controls. When cholesterol saturation indices (CSIs) are calculated according to published tables (8) for taurocholatecontaining model bile, gallbladder biles of male and female transgenic mice, but not littermate controls, are found to be supersaturated, which would ordinarily indicate that the bile is not homogeneous but includes cholesterol precipitates in one or two separate phases of cholesterol crystals and/or liquid crystals. Curiously, the authors did not detect cholesterol gallstones or even cholesterol monohydrate crystals when bile was examined under a polarizing light microscope. Bearing in mind that gallbladder biles from $A B C G 5 / A B C G 8$ transgenic mice are appreciably more cholesterol-enriched than those found in gallstone-susceptible mice fed a lithogenic diet containing cholesterol and cholic acid (9), why do these animals not form cholesterol gallstones? The bile salt pool of mice, as opposed to humans, is composed of large proportions of hydrophilic bile salts (predominantly tauro- $\beta$-muricholate), which can reach levels varying from $40 \%$ to $70 \%(9,10)$; in the current study there was an equimolar ratio of cholic and muricholic acids. Increased hydrophilicity of bile salts in bile leads to decreased micellar cholesterol solubility and marked alterations of the boundaries of the multi-phase zones above the micellar phase (11).

Figure 1 displays the consequences of this physical chemical property of mouse bile. Not only is the micellar zone appreciably reduced compared to that of more hydrophobic bile salts $(8$, 11), but the physical states and the crystallization pathways in the phase diagram are moved considerably to the left $(11,12)$. Bile samples from male and female littermate controls are slightly supersaturated with cholesterol and fall above the micellar phase (Figure 1). In contrast, the high cholesterol levels in bile from $A B C G 5 / A B C G 8$ transgenic 
mice result in an upward shift of the relative lipid compositions toward the cholesterol apex of the triangle. We estimate a corrected CSI for female transgenic mice of 9.0 and for male transgenic mice of 7.0 when adjusted for the low micellar cholesterol solubility in this bile salt composition. However, because of the left shift of phase boundaries with increased hydrophilicity (Figure 1 ), bile compositions from transgenic mice are close to a zone of the phase diagram where a solid cholesterol crystalline phase is a forbidden transition at equilibrium (11).

The localization of relative lipid compositions near this two-phase zone of mixed micelles and liquid crystals (Figure 1) may explain the failure of a solid phase to separate over the time course of these experiments. In addition, the authors (2) note an "opaque" macroscopic appearance of gallbladder bile in transgenic animals, typical of a liquid crystalline phase in bile that floats on the micellar phase due to its lower specific density. Because a narrow, 30.5 gauge hypodermic needle was used to aspirate gallbladder bile (2), it is possible that only multilamellar liquid crystals were removed for analysis and that aggregated cholesterol crystals and microscopic stones indeed existed in the gallbladder, as our phase analysis would predict, but that they were left behind (Figure 1). Feeding small amounts of cholic acid to the ABCG5/ABCG8 transgenic animals would replace much of the biliary tauro- $\beta$-muricholate with taurocholate (12), leading to a right-shift of the crystallization zones in the phase diagram
(11), and accordingly would lead to rapid nucleation of cholesterol monohydrate crystals and formation of macroscopic cholesterol gallstones visible through the transparent murine gallbladder wall.

\section{Phospholipids lag behind biliary cholesterol secretion}

In the lithogenic state, increased biliary cholesterol levels are coupled to markedly increased biliary lecithin levels in that relative lipid compositions of cholesterol supersaturated bile do not shift toward the cholesterol apex of the phase diagram, but towards a point one third to midway along the phospholipid axis (9). Interestingly, although biliary lecithin levels in transgenic mice were increased, a line connecting relative biliary lipid

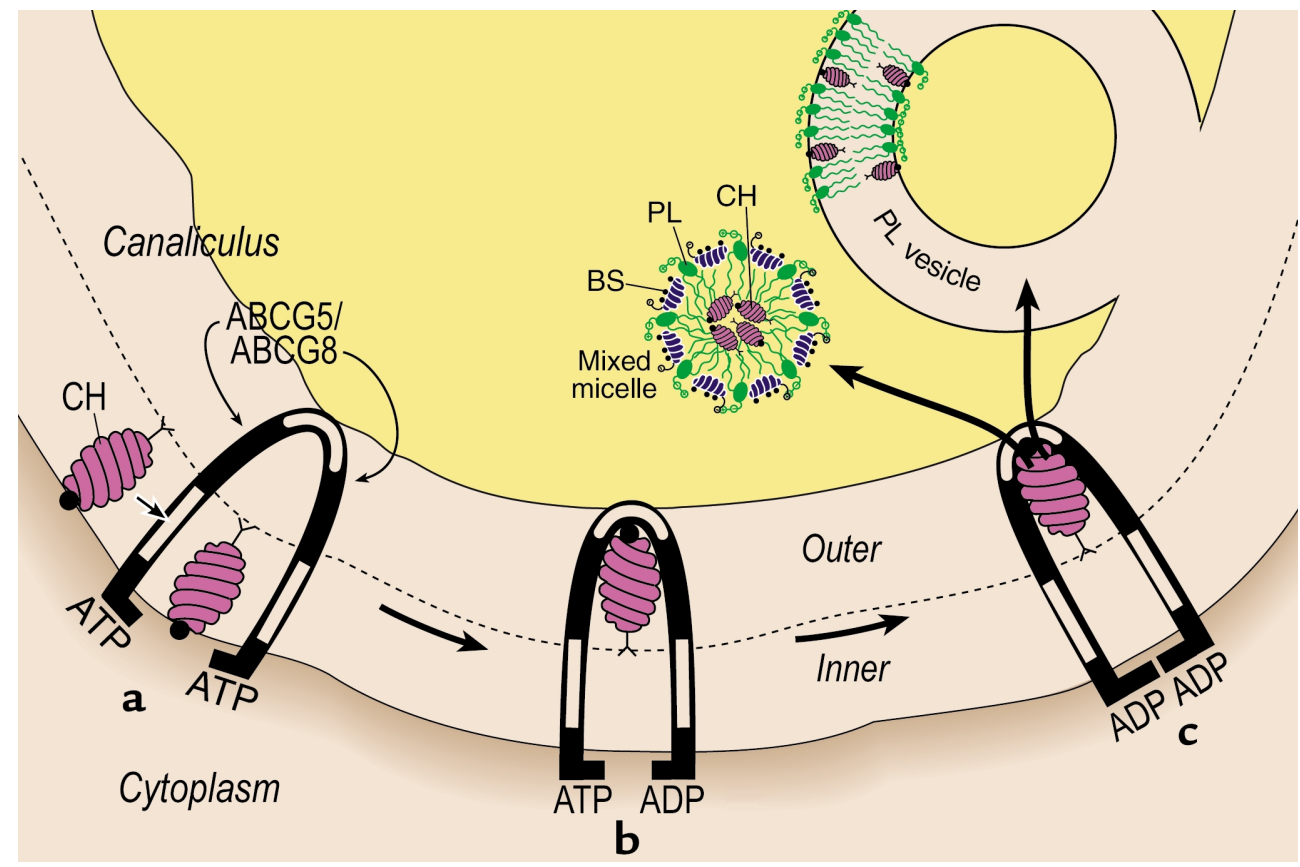

Figure 2

A hypothetical transporter model for transmembrane "flipping" of cholesterol molecules by the human ABCG5/ABCG8 heterodimer. The figure is based on the x-ray crystallogrophic structure of MsbA, the lipid flippase from Escherichia coli, which is also composed of 2 half-transporters, but forms a homodimer (19). The organization of the ABCG5/ABCG8 heterodimer is such that the two subunits, each containing 6 transmembrane $\alpha$-helices, physically interact to form a truncated cone within the membrane lipid bilayer. For thermodynamic reasons, membrane lipid is likely present within as well as outside this structure. It is believed that transport is initiated (a) upon binding ATP at a nucleotide-binding domain located on the cytoplasmic face of the half-transporters (19). Through side entrances of the chamber, the transporter captures a cholesterol molecule from the inner leaflet of the canalicular membrane (a). The transport dynamics then require that ATP-hydrolysis induce a conformational change forcing the two half-transporters to close partially at the cytoplasmic side (b), rendering the cholesterol molecule thermodynamically uncomfortable on the inner leaflet. This induces "flip-flop" of cholesterol across the membrane lipid within the transporter, bringing the sterol and its hydroxyl function to lie in a correct orientation at the lipid-water interface of the outer membrane leaflet (19). With continued ATP hydrolysis (c), the cholesterol molecules are expelled into nascent bile from the exoplasmic face of the transporter as monomers (solubility $10^{-8} \mathrm{M}$ ), to diffuse to solubilization sites within bile salt micelles and phospholipid vesicles in the canalicular space (10, 21). This transporter model is based on $\mathrm{x}$-ray crystallographic work of Chang and Roth (19) and incorporates theoretical suggestions of Higgins and Linton (25). Unilamellar vesicle and mixed micelle models, with close-packing of the lipids, are based on cryophotoelectromicrographs of Crawford et al. (21) and molecular dynamics simulations of Marrink and Mark (22), respectively. The molecular models are drawn to conform to space-filling dimensions for cholesterol ( $\mathrm{CH}$ ), phospholipid ( $\mathrm{PL}$ ), and bile salt ( $\mathrm{BS}$ ). Filled circles, hydroxyl functions; filled ovals, glycerol; wavy lines, acyl chains; inscribed positive signs, cationic groups; inscribed negative signs, anionic groups; large scalloped cross-hatched ovals, saturated (BS) or unsaturated $(\mathrm{CH})$ steroid nucleus. 
compositions of littermates through the transgenic biles extrapolates to the cholesterol apex of the triangle. This analysis (Figure 1) suggests that secretion of cholesterol and lecithin into the canalicular bile has become uncoupled and supports the notion that the phospholipid content in the bile limits the rate of cholesterol secretion in $A B C G 5 / A B C G 8$ transgenic mice. Hence, it appears that bile in these animals does not exactly mimic the lithogenic state in cholesterol gallstone-susceptible mice (9). This conclusion is further substantiated by the observation that cholesterol levels in gallbladder bile are similar in transgenic animals with high or low copy numbers of the ABCG5 and ABCG8 genes (2). Therefore, cholesterol levels high enough to lead to supersaturated bile may be achieved by overexpressing the two half-transporters to a moderate extent, within the range that could be caused by regulatory region polymorphisms in $A B C G 5$ and $A B C G 8$. Indeed, in a recent quantitative trait locus cross of two inbred mouse strains $(13,14)$, we identified the locus on murine chromosome 17 that includes $A b c g 5$ and $A b c g 8$ as a region that is associated with cholesterol gallstone susceptibility (15). Moreover, the high biliary cholesterol levels in the susceptible parental strain correlate with high hepatic expression of these two gene products (H. Wittenburg, M.A. Lyons, B. Paigen, M.C. Carey, 2002, unpublished observations). The combined results of the analysis of human ABCG5/ABCG8 function in mice and the identification of their murine homologs as strong positional candidate genes for cholesterol gallstone formation warrant testing of $A B C G 5$ and $A B C G 8$ in human cholelithiasis patients, as Yu et al. also suggest (2).

Finally, transgenic mice not only exhibited the expected marked increase in fecal neutral sterol secretion, but females of this strain also significantly increased their excretion of fat. Based on biliary lipid analyses, the ratio of cholesterol to lecithin in the phase-separated vesicles in bile approximates 1.4:1 for females and 1:1 for males. Such levels of cholesterol to lecithin in multilamellar vesicles result in a semicrystalline physical state (16) in which the phospholipids are resistant to hydrolysis by pancreatic phospholi- pase $A_{2}$. For the same reason, high cholesterol levels with "acid soaps" and monoglycerides, both liquid-crystalline hydrolytic products of dietary triglyceride digestion, would greatly retard their bile salt micellar solubilization and restrict their efficient absorption. This phenomenon is well known in the case of sphingomyelin, a minor biliary but major dietary phospholipid. Not only does sphingomyelin curtail cholesterol absorption $(17,18)$, but high cholesterol levels also prevent sphingomyelin's hydrolysis and absorption (17).

\section{Proposed molecular model for functioning of the ABCG5/ABCG8 heterodimer}

Only recently has the high resolution structure of an ABC transporter been solved by x-ray crystallography (19). In this case, the Escherichia coli lipid flippase MsbA (closely related to mammalian multidrug resistance (MDR) proteins) forms a homodimer. By analogy to the proposed function of MsbA (19), we suggest that the ABCG5/ ABCG8 heterodimer acts by shuttling cholesterol from the inner leaflet of the canalicular membrane through a chamber formed by the two half-transporters. Following ATP binding and hydrolysis, the complex undergoes a conformational change, flipping a cholesterol molecule into the outer membrane leaflet in a configuration that favors its release into the canalicular space. Analysis of bile from mice made deficient in the canalicular phospholipid transporter MDR2 (10) has revealed only minimal cholesterol secretion into bile; this poor secretion phenotype can be partially reversed by intravenous taurocholate infusion or completely reversed by infusion of the more hydrophobic bile salt taurodeoxycholate (20). In $M d r 2^{-/+}$heterozygotes biliary phospholipid levels are $50 \%$ of controls, but cholesterol secretion is normal $(10,20)$, consistent with a limiting physical chemical or indirect role for MDR2 in the cholesterol transport process. These data suggest that acceptor macromolecules must be present on the luminal side of the canalicular membrane in order to facilitate cholesterol's secretion into bile, since aqueous solubility of the sterol is minimal $\left(\approx 10^{-8} \mathrm{M}\right)$. Therefore, we propose that selfaggregated biliary phospholipids and bile salts function as cholesterol acceptors within the canalicular space, forming cholesterol-rich vesicles (21) and mixed micelles $(20,22)$, respectively (Figure 2).

The present studies on cellular trafficking and in vivo functioning of ABCG5 and ABCG8 greatly advance our understanding of cholesterol and phytosterol transport into bile. We are taught in the first paper (1) that the heterodimeric units are physically and biologically inseparable in their journey to the apical membrane; in the second (2), we witness the molecular, biological, biochemical, and biophysical results of flooding bile with enormous amounts of excess cholesterol that is, in part, prevented from being reabsorbed. Still, it remains to be explained why the secretion into bile of cholesterol and other sterols is reduced by only $50 \%$ in patients with sitosterolemia. The residual sterol secretion suggests that other transport mechanisms operate in parallel to mediate canalicular capture of sterols and their biliary secretion (23). The ability to abolish Abcg5/Abcg8 function by knocking out either or both of the corresponding genes should allow for the systematic study of this activity in the mouse.

1. Graf, G.A., et al. 2002. Coexpression of ATP bind ing cassette proteins ABCG5 and ABCG8 permits their transport to the apical surface. J. Clin. Invest. 110:659-669. doi:10.1172/JCI200216000.

2. Yu, L., et al. 2002. Overexpression of ABCG5 and ABCG8 promotes biliary cholesterol secretion and reduces fractional absorption of dietary cholesterol. J. Clin. Invest. 110:671-680 doi:10.1172/JCI200216001.

3. Berge, K.E., et al. 2000. Accumulation of dietary cholesterol in sitosterolemia caused by mutations in adjacent $\mathrm{ABC}$ transporters. Science. 290:1771-1775.

4. Lee, M.H., et al. 2001. Identification of a gene, ABCG5, important in the regulation of dietary cholesterol absorption. Nat. Genet. 27:79-83.

5. Salen, G., et al. 1992. Sitosterolemia. J. Lipid Res. 33:945-955.

6. Björkhem, I., Boberg, K., and Leitersdorf, E. 2001 Inborn errors in bile acid biosynthesis and storage of sterols other than cholesterol. In The Metabolic and Molecular Basis of Inherited Diseases. C. Scriver, A. Beaudet, W. Sly, and D. Valle, editors. McGraw-Hill. New York, New York, USA. 2961-2988.

7. Lu, K., et al. 2001. Two genes that map to the STSL locus cause sitosterolemia: genomic struc ture and spectrum of mutations involving sterolin-1 and sterolin-2, encoded by ABCG5 and ABCG8, respectively. Am. J. Hum. Genet. 69:278-290.

8. Carey, M.C. 1978. Critical tables for calculating the cholesterol saturation of native bile. J. Lipid Res. 19:945-955.

9. Wang, D.Q., Paigen, B., and Carey, M.C. 1997. Phenotypic characterization of Lith genes that determine susceptibility to cholesterol cholelithiasis in inbred mice: physical-chemistry of gall bladder bile. J. Lipid Res. 38:1395-1411. 
10. Oude Elferink, R.P., et al. 1995. Regulation of biliary lipid secretion by mdr2 P-glycoprotein in the mouse. J. Clin. Invest. 95:31-38.

11. Wang, D.Q., and Carey, M.C. 1996. Complete mapping of crystallization pathways during cholesterol precipitation from model bile: influence of physical-chemical variables of pathophysiologic relevance and identification of a stable liquid crystalline state in cold, dilute and hydrophilic bile salt-containing systems. J. Lipid Res. 37:606-630.

12. Wang, D.Q., Lammert, F., Cohen, D.E., Paigen, B., and Carey, M.C. 1999. Cholic acid aids absorption, biliary secretion, and phase transitions of cholesterol in murine cholelithogenesis. Am. J. Physiol. 276:G751-G760.

13. Lammert, F., Carey, M.C., and Paigen, B. 2002. Chromosomal organization of candidate genes involved in cholesterol gallstone formation: a murine "gallstone map". Gastroenterology. 120:221-238.

14. Wittenburg, H., Lyons, M.A., Paigen, B., and Carey, M.C. 2002. Mapping cholesterol gallstone susceptibility (Lith) genes in inbred mice. Dig. Liver Dis. In press.

15. Wittenburg, H., Lyons, M.A., Li, R., Carey, M.C., and Paigen, B. 2002. New cholesterol gallstone susceptibility $($ Lith) loci with attractive positiona candidate genes in an intercross of PERA/Ei and I/LnJ strains of mice. Gastroenterology. 122:A543. (Abstr.)

16. Phillips, M.C. 1990. Cholesterol packing, crystallization and exchange properties in phosphatidylcholine vesicle systems. Hepatology. 12:75S-82S.

17. Nyberg, L., Duan, R., and Nilsson, A.A. 2000. A mutual inhibitory effect on absorption of sphingomyelin and cholesterol. J. Nutr. Biochem. 11:244-249.

18. Eckhardt, E.R.M., Wang, D.Q., Donovan, J.M., and Carey, M.C. 2002. Dietary sphingomyelin suppresses intestinal cholesterol absorption by decreasing thermodynamic activity of cholesterol monomers. Gastroenterology. 122:948-956.

19. Chang, G., and Roth, C.B. 2001. Structure of MsbA from E. coli: a homolog of the multidrug resistance ATP binding cassette (ABC) transporters. Science. 293:1793-1800.

20. Oude Elferink, R.P., et al. 1996. Uncoupling of biliary phospholipid and cholesterol secretion in mice with reduced expression of mdr2 P-glycoprotein. J. Lipid Res. 37:1065-1075.

21. Crawford, J.M., et al. 1995. Imaging biliary lipid secretion in the rat: ultrastructural evidence for vesiculation of the hepatocyte canalicular membrane. J. Lipid Res. 36:2147-2163.

22. Marrink, S.J., and Mark, A.E. 2002. Molecular dynamics simulations of mixed micelles modeling human bile. Biochemistry. 41:5375-5382.

23. van Erpecum, K.J., and Carey, M.C. 1997. Influence of bile salts on molecular interactions between sphingomyelin and cholesterol: relevance to bile formation and stability. Biochim. Biophys. Acta. 1345:269-282.

24. Montet, J.-C., et al. 1987. $\beta$-muricholic acid, potentiometric and cholesterol-dissolving properties. Biochim. Biophys. Acta. 918:1-10.

25. Higgins, C.F., and Linton, K.J. 2001. The xyz of ABC transporters. Science. 293:1782-1784. 